\title{
Estudiantes Sordos señantes: un botón de muestra de la importancia de participar desde la infancia
}

\author{
Miriam Viridiana Verástegui Juárez ${ }^{1}$ \\ Universidad Autónoma Metropolitana-Xochimilco
}

\section{Artículo Científico}

Material original autorizado para su primera publicación en el Journal de Ciencias Sociales, Revista Académica de la Facultad de Ciencias Sociales de la Universidad de Palermo.

\section{Recepción: 12-03-2018}

Aceptación: 04-07-2018

Resumen: Esta investigación presenta la importancia de la participación de los alumnos Sordos Señantes, tomando como botón de muestra a dos universidades, una tecnológica y otra politécnica, por ser estas las que en México abren sus puertas a la inclusión educativa de esta población. El marco de referencia que se tomó para el trabajo de campo es la participación como proceso fundamental para la inclusión efectiva. Los resultados, aunque alentadores en todas las acciones que se llevan a cabo para que las personas sordas tengan acceso a los contenidos académicos de sus carreras, dejan una amplia línea de intervención en la vinculación con otras universidades y en la promoción de las ideas y proyectos que los alumnos y alumnas han desarrollado para que estos modelos puedan implementarse en otras universidades. Actualmente, los estudiantes no son informados ni consultados sobre decisiones que afectan al sistema escolar. Esto es así no sólo en los niveles de educación básica sino también en la educación media y hasta la superior. La literatura apunta a que la participación de los y las alumnas en sus centros es fundamental para construir escuelas democráticas. Será entonces vital que, en todos los niveles educativos, las voces de los estudiantes sean escuchadas para que a través de su experiencia se puedan comenzar a delinear acciones y líneas de trabajo que permitan el ejercicio del derecho a una educación inclusiva para que los alumnos y alumnas Sordas se vivan como agentes de cambio, aportando sus conocimientos y experiencias para promover una sociedad más justa e inclusiva.

Palabras clave: sordos; participación; inclusión; lengua de señas mexicana.

\footnotetext{
${ }^{1}$ Lic. en Psicología, con Mención honorífica, por la Universidad Nacional Autónoma de México; Maestra en Desarrollo y Planeación de la educación por la Universidad Autónoma Metropolitana -Xochimilco. Se ha capacitado continuamente sobre temas referentes a la discapacidad desde miradas socio-antropológicas, sexualidad y discapacidad y metodologías para la adquisición de la lectura y la escritura en personas con discapacidad auditiva e intelectual.

Correo electrónico: verasteguijv@gmail.com
} 


\title{
Deaf Signers Students: a Button Showing The Importance of Participating Since Childhood
}

\begin{abstract}
This investigation introduces the importance of Deaf student's participation having as reference frame two Universities; one of them, a Technological University and the other one, a Polytechnic University. These universities were chosen due to the fact that they are open to inclusive education for these population. The reference frame taken for the field work, is participation as fundamental process for effective inclusion. Even when the results for actions taking place for deaf people in order to have access to academic contents of their career are encouraging, they leave a huge space for intervention in the link with other universities and in the promotion of ideas and projects that students have developed so that these models be implemented in other universities. To this day, students are not consulted about decisions concerning the school system. This is not just happening in elementary school but in middle and high school. Different publications argue about the importance of student participation as fundamental to build democratic schools. It will be absolutely necessary on all education levels that student voices be heard, so that through their experience, actions and plans may be traced to allow the possibility of exercising the right to inclusive education, so that deaf students experience themselves as agents of change, providing their experiences and knowledge to promote a more inclusive and fair society.
\end{abstract}

Keywords: deaf; participation; inclusion; mexican sign language.

\section{Introducción}

El presente trabajo pretende hacer una aportación descriptiva acerca de la participación que tienen los estudiantes Sordos usuarios de la Lengua de Señas Mexicana LSM- en los modelos de inclusión que existen en México, dentro de las Universidades Tecnológicas y Politécnicas (UTyP). Si bien no hay una sola definición de inclusión educativa, distintos autores coinciden en que la participación es una vía para que se logre el cometido de este proceso, por ello se dará especial atención a este tema. A su vez, muestra la oportunidad de llevar las experiencias de la participación de niños, niñas y jóvenes a las universidades que se están decidiendo a dar espacios para que las personas con discapacidad auditiva puedan ejercer su derecho a una educación inclusiva que tome en cuenta su voz.

Para este estudio es vital garantizar la plena inclusión a la educación de esta población ya que, como menciona Tomasevski (2002, citado en Ruíz y Cruz, 2013), la educación permite a los ciudadanos garantizar el disfrute de todos los derechos y libertades. 
De acuerdo con Gómez (2014) se han encontrado diversas universidades a lo largo del país con experiencias en inclusión educativa para sordos señantes. No obstante, la falta de sistematización y profesionalización de los modelos hace que sean casos aislados. Debido a que en México no hay políticas claras en torno a la inclusión de personas con discapacidad en educación superior, la investigación de este fenómeno puede aportar información para conocer la experiencia de los alumnos sordos señantes y para posibilitar formas de implementación que tomen en cuenta la participación de los Sordos.

Torres (1995) escribe sobre la importancia de la participación de todos los actores en la escuela, destacando que no es suficiente con acceder a ella: el derecho a la educación implica el derecho a participar. Actualmente, los estudiantes no son informados ni consultados sobre decisiones que afectan al sistema escolar y mucho menos sobre la política educativa. Esto es así no solo en los niveles inferiores del sistema, también en la educación media y hasta la superior. Agrega que la participación, en educación, no es un agregado o una opción: es condición indispensable del hecho educativo (el aprendizaje es activo y participativo, o no es tal). No entender esto lleva una y otra vez al no-aprendizaje, a la falta de adecuación de políticas y programas a la realidad, a la incomprensión y resistencia frente a las propuestas de cambio, entre otras.

El objetivo es analizar cuál es la participación de los estudiantes sordos en estos modelos, ya que es a través de ellos y su experiencia que se pueden comenzar a delinear acciones y líneas de trabajo para que puedan ejercer plenamente su derecho a la educación.

Se pretende promover cada vez más no solo desde la educación superior, sino desde la básica, escuchar la voz de los niños, niñas y jóvenes en la escuela para tener un acercamiento a la investigación participativa y emancipatoria, como la llaman Oliver y Barnes (1992). De este modo los estudiantes se pueden sentir agentes de cambio con la capacidad de aportar no únicamente para su bienestar, sino para las generaciones que les preceden, elemento sumamente importante para abonar a las prácticas inclusivas de los y las estudiantes en esta condición.

\section{La participación de los estudiantes Sordos señantes en las UTyP ¿Por qué y para qué?}

Una vez hecho el recorrido de artículos que hablan sobre participación y discapacidad queda claro que cada uno focaliza el término desde distintos ámbitos y que, en ocasiones, incluso se evidencia el concepto, es decir, que presuponen una definición clara de él. Por ejemplo, en una investigación que tiene como objetivo la participación comunitaria de las personas con discapacidad, obvian el concepto, aunque proponen acciones ligadas a él: la 
vinculación en la familia, involucramiento en actividades escolares, además de experiencias y formas de interacción social (Rodríguez, Alvarado, y Moreno, 2007). Otra investigación sobre participación escolar vincula el término con la actividad en el salón de clases, excursiones y eventos escolares, equipos y clubs, reunirse con compañeros fuera del horario escolar y roles especiales en la escuela. No obstante, tampoco define el concepto (Coster, Law, Bedell, Liljenquist, Kao, Khetani, y Teplicky, 2012). Finalmente, en inclusión educativa se percibe la participación como la calidad de las experiencias cuando los alumnos se encuentran en la escuela y la satisfacción ante las mismas (López, López y Llorent, 2009).

El párrafo anterior da cuenta de que en general se toma a la participación como ser parte de, es decir, estar presente en las actividades y en los grupos sociales. No se descarta que esto sea justamente participación. Sin embargo, lo que se pretende decir aquí es que no es solo eso. Desde teorías que analizan la participación ciudadana y la de los niños y niñas, por ejemplo, lo anterior quedaría como el nivel más simple de esta acción, proponiendo que la esencia es la toma de decisiones y la influencia que cada persona pueda tener en sus diferentes grupos de pertenencia.

Como primer aspecto en la definición se resalta que la participación es compartir decisiones que afectan directamente a la persona o a su comunidad (Corona, y Morfín, 2001), como interacción humana y esfuerzo para la consecución de un cambio o bien común (Casilla e Inciarte, 2004). La segunda parte podría ligarse a cómo hacer efectivo que cada miembro de la comunidad -cualquiera que esta sea- pueda ser agente de cambio y formar parte de la toma de decisiones. Distintos autores coinciden en que la diversidad de características individuales enriquece lo comunitario, por lo que promover que cada miembro del grupo sea capaz de brindar sus potencialidades individuales es decisivo para lograr el bien común. En este sentido, valorar la diversidad antecede al proceso de participación (Corona, y Morfín, 2001).

Se evidencia hasta aquí la relación que existe entre estos dos primeros puntos del concepto y la situación que envuelve a la inclusión educativa de las personas con discapacidad. Se esperaría que la postura de las instituciones fuera valorar la diversidad y promover que los alumnos Sordos tengan la oportunidad de ser estudiantes que decidan sobre lo que les afecta directamente en su institución. Se sabe que hay distintos temas en los que las y los alumnos pueden participar dentro de una institución, estén estos en situación de discapacidad o no. Sin embargo, este estudio centra su atención en la participación que tienen en el modelo de inclusión. Esto responde al impacto que este tiene en su paso por la universidad. Se postula que es al menos en este ámbito donde es imperante que se tome en cuenta su opinión y que puedan ser capaces de intervenir en las decisiones para que puedan aprovechar al máximo su presencia en la universidad. Si logran, como alumnos, ser parte de 
este proceso, irán construyendo una visión de sí mismos como personas importantes dentro de la comunidad donde se encuentran. También se espera que este tipo de prácticas les permita tener más autonomía y confianza en sus habilidades.

Finalmente, se dice de la participación que es un proceso que puede habilitar a los excluidos a ser deliberadamente incluidos en el futuro para que puedan compartir los beneficios de los que tienen todo (Arnstein, 1969). Además, potencia la incidencia de las voces que han sido silenciadas por ser personas con discapacidad, reconociendo sus aportes positivos y constructivos en la comunidad (Yarza, Rojas y López, 2014). Así pues, se puntualizan los tres aspectos más importantes que definirían la participación de los alumnos Sordos en los modelos de inclusión:

1) Involucrarlos en la toma de decisiones sobre las acciones que serán tomadas para su inclusión educativa. No se trata sólo de consulta sino de injerencia.

2) Valorar la diversidad y tener apertura a los aportes que pueda hacer a la comunidad. Es prudente que sean parte del proceso de toma de decisiones, desde su perspectiva. Como personas con discapacidad auditiva pueden aportar ideas para que les resulte más enriquecedor su paso por la universidad.

3) Reconocer que las prácticas y espacios participativos los llevan a valorarse positivamente como agentes de cambio, beneficiándose de espacios que en general les han sido negados por su condición en discapacidad.

Para responder a la pregunta de por qué participar, el análisis puede comenzar desde la relación que hay entre esta acción y democracia. La importancia de que las personas puedan incidir en las comunidades de las que son partícipes corresponde a que participar no sea un fin en sí mismo, puesto que está vinculada al fin último de construir la democracia (Hart, citado en Expósito, 2014). Así, se entiende que la participación dentro de las escuelas y en caso particular dentro de las UTyP, no solo abonará a que los alumnos Sordos sean parte de ella; también coadyuvará a que estas personas puedan tener una postura de ciudadanía, aspecto que se resalta desde la Convención sobre los Derechos de las Personas con Discapacidad, para poder mitigar los años en que han estado relegados de la vida política y social.

Así, uno de los principios generales de esta Convención es: La participación e inclusión plenas y efectivas de las personas con discapacidad en la sociedad, tomando en cuenta:

el valor de las contribuciones que realizan y pueden realizar las personas con discapacidad al bienestar general y a la diversidad de sus comunidades, y que la promoción del pleno goce de los derechos humanos y las libertades fundamentales por las personas con discapacidad y de su plena participación tendrán como resultado un mayor sentido de pertenencia de estas personas y avances significativos en el desarrollo económico, social y humano de la sociedad 
y en la erradicación de la pobreza" (Convención sobre los derechos de las personas con discapacidad, 2006, p.2).

A partir del hilo conductor del apartado anterior y como respuesta a la pregunta de para qué participar, se puede decir que una de las cualidades que se observan en los estudiantes gracias a transitar por ambientes participativos según Mager y Nowak (citado en Expósito, 2014) son las habilidades de comunicación, la toma de decisiones, la resolución de problemas y habilidades interpersonales y sociales. Esto quiere decir que, si una persona con discapacidad está en una condición de desventaja por su condición biológica y en el caso de los Sordos por no hablar español, es menester desarrollar habilidades comunicativas, resolución de problemas y trabajo en equipo para poder promover seguridad en ellos mismos y en sus potencialidades.

Dentro de las habilidades en comunicación y relaciones interpersonales, diversas investigaciones han demostrado que, cuando los jóvenes participan, tienen más capacidad de toma de decisiones de manera personal y grupal, identificación y solución de problemas, capacidad para discutir con los demás y para llegar a acuerdos (Corona, Y. y Morfín, M., 2001). Lo anterior puede derivar en que además de generar esas habilidades, puedan ser conscientes de la diversidad, la de ellos como personas con discapacidad y también la de los demás. Hart (1993) explica que la participación fomenta el respeto hacia los otros y hacia su forma de expresión, situación que converge con el valor a la diversidad.

"Hay estudios que demuestran que las familias con buenos recursos tienden a valorar la independencia y la autonomía mientras que las de bajos ingresos valoran la obediencia de los niños" (Hart, 1993, p.40). Estos son puntos que no se deben pasar por alto en las instituciones de educación superior a la hora de diseñar las acciones de inclusión para las personas con discapacidad, ya que, si en general en sus familias y en la educación básica y media no se ha promovido la participación de los alumnos Sordos, es muy probable que cuando lleguen a la universidad no sean ellos los que pidan participar. Así, la escuela, sabiendo la riqueza que pueden brindar los espacios participativos, podrá considerar su promoción. Finalmente, la participación de las personas con discapacidad está sustentada en que ellos, al ser los usuarios de los servicios que brindan las UTyP, son los que mejor saben si les son funcionales o no, y si podrían mejorarlos en algunos aspectos para que puedan ser más fructíferos. Si las personas con discapacidad no están involucradas en los procesos donde se deciden las herramientas que les proporcionarán, puede que la inversión en estas sean soluciones que den pocos o nulos resultados. (Joly, 2011).

\section{Dimensiones de la participación}


Una vez que se ha definido el concepto de participación y que se ha justificado su promoción en las UTyP para los alumnos con discapacidad auditiva, se procede a esclarecer las dimensiones de participación que fueron tomadas en cuenta para esta investigación. La importancia que los teóricos de la participación le han dado deriva, en parte, de que se ha encontrado que el proceso no siempre se da de forma genuina, en algunas ocasiones se hace para cumplir ciertas cuotas, para mejorar la imagen política, e incluso por la propia concepción que se tiene del término participar.

Arnstein (1969) alerta sobre la diferencia que hay entre el ritual de la participación y el poder real de incidir en los resultados del proceso. Se habla de una simulación, por ejemplo, en algunos foros cuando se invita a niños a participar y es un adulto quien escribe el discurso y le enseña a moverse como un político. El participante no es más que una marioneta que aprende de memoria algo que quizá no entiende y que no generó a través de su experiencia. Por ello el autor antes mencionado diseñó la escalera de la participación, que ha retomado Hart (1993) y la ha adaptado para la participación de los niños. La escalera cuenta con ocho peldaños y en orden ascendente van de la manipulación hasta el control ciudadano.

1 y 2: manipulación y utilización: Estos dos escalones describen los niveles de no participación o de participación simulada. Su objetivo no es promover que las personas tengan voto en los proyectos, sino que se les dirá lo que deben hacer y qué es lo mejor para ellas. En las personas con discapacidad auditiva, por ejemplo, se les puede invitar a que sean parte de terapias de rehabilitación, cuando jamás se les invitó a participar para que ellos pusieran sobre la mesa sus necesidades (Arnstein, 1969).

3, 4 y 5: Información, consulta y cooperación; se encuentran dentro del tokenismo, que se define como un acto positivo relativamente pequeño o trivial, se emplean minorías en aspectos no relevantes para que se sientan incluidos y no tengan necesidad de ser convocados o de participar en procesos más importantes que competan a su comunidad (Hogg, y Vaughan, 2010). Bajo estas perspectivas los ciudadanos escuchan, pero también se hacen escuchar, aunque tendrán una falta de poder para asegurar que su punto de vista sea promovido en el poder. En el escalón 5 hay una pequeña diferencia cuando se habla de cooperación, se hace referencia a que algunos podrán no solo dar su punto de vista, sino que además podrán aconsejar y proponer, pero la decisión seguirá estando en manos del que tiene el poder (Arnstein, 1969).

6, 7 y 8: Estos escalones refieren a la participación efectiva y el grado irá aumentando ascendentemente. El primero se refiere a la alianza estratégica, que permite negociar medidas compensatorias con el poder tradicional. Es decir, que ambas partes -poder y grupose involucran en la toma de decisiones. La alianza estratégica puede funcionar de mejor manera cuando hay una organización efectiva en la comunidad donde se discute lo que es 
mejor para ella. El 7 refiere a delegar poder a algunos miembros del grupo, la diferencia con el anterior es que tienen más votos dentro del comité de toma de decisiones y así aseguran que su opinión sea tomada en cuenta. Finalmente, el ocho, siendo este el último, se refiere al control ciudadano que es cuando el grupo toma mayores decisiones e incluso puede tomar completamente el poder de las intervenciones que le afectan. El ejemplo más claro de esta situación son los grupos de autogestión (Arnstein, 1969).

Aunque la categorización presentada anteriormente no habla de por qué en algunas ocasiones los grupos se quedan únicamente en los niveles más simples de participación, hay estudios que dan algunos datos de los que podríamos generar inferencias. El mismo Arnstein (1969) propone que las barreras pueden estar colocadas en ambos lados; por la parte de los tomadores de decisiones y, en el caso de la discapacidad, se puede plantear la posibilidad del sentimiento paternalista, es decir, que se piensa que las personas con discapacidad no están preparadas para tomar decisiones o que no saben qué es lo mejor para ellas. Por el lado de las personas con discapacidad se espera que, si durante su experiencia de vida se ha fomentado poco la participación de los niveles más altos, que no han experimentado la negociación para alcanzar objetivos que deseen o que les sean favorables, más aún, si no han generado espacios de diálogo con sus pares para alcanzar metas que favorezcan a su comunidad, ni mucho menos tomado el control en la toma de decisiones de su propia vida y del grupo al que se sientan pertenecientes, entonces tendrán dificultad en la organización, la representatividad y posible alienación.

Para entender de forma más clara los elementos que pueden estar en juego a la hora de participar en los diferentes niveles propuestos, se plantea que el proceso de toma de decisiones en un grupo tiene como objetivo el bien común y que juega con ciertas acciones; es decir, actos que son intencionales y forman parte de un interés o necesidad, a través de la cual se pretende incidir. Lo anterior se vincula con la interacción, que se manifiesta en la interrelación del ser humano con los otros seres que lo rodean. A través de esta se generan cambios bidireccionales, de las personas hacia los sistemas y viceversa. Con ella finalmente se realiza un cambio, que es producto de una deliberación sobre una situación que se quiere distinta a como se encuentra actualmente y que pretende ser más conveniente para el actor, el colectivo o el contexto (Castilla e Inciarte, 2004).

Ahora bien, para que se generen estas acciones se precisa que la persona y el grupo que participa tengan conocimientos. Dentro de estos se encuentra la formación, que incluye las posibilidades y oportunidades para estar informado e informar sobre lo que interesa a la organización o lo que necesita para tomar decisiones; también está la información que incluye los conceptos o significados con los que trabaja el grupo para participar. Finalmente, todo lo anterior está cargado de valores, premisas o creencias, esto es lo que da fuerza a la decisión 
de involucrarse en una acción (Ídem). Esta decisión de involucrarse en una acción participativa está relacionada a su vez con la eficacia externa e interna (Niemi, Craig, y Mattei, citado en Expósito, 2015). La interna se refiere a creer en la capacidad que se tiene para que, a través de la participación, se pueda generar un cambio significativo, mientras que la externa, se refiere al grado de confianza que depositan en las autoridades escolares para que atiendan las inquietudes que les presenten.

Así, la eficacia interna en los alumnos Sordos en las UTyP converge con su autoconcepto, que como veíamos en párrafos anteriores está ligado a la forma en que conciben sus características y potencialidades, mientras más participen es más probable que promuevan cambios que les beneficien, de ahí la importancia de que estas experiencias participativas formen parte de toda su trayectoria escolar, desde la básica hasta la superior (Expósito, 2015).

Ahora bien, el concepto que tengan de las autoridades escolares depende de los espacios que abran para la participación de los estudiantes y de las herramientas que den información, promoción, valor a la participación para que estos decidan participar (Ídem). Como se observa, se ha regresado a hablar de algunas de las características de la participación con las que se respondió anteriormente la pregunta ¿para qué participar? Esto se debe a que el proceso de participación no es lineal, es una espiral que va entrelazando las prácticas democráticas y, a su vez, va desarrollando habilidades tanto en la institución como en los alumnos involucrados. Entre más prácticas haya, las habilidades van cambiando, lo que lleva a mejor desarrollo de ambas partes en la interacción.

Es así como se concluye con la premisa con la que comenzó el capítulo: la participación está íntimamente relacionada con la inclusión educativa, no solo en las universidades sino en todos los niveles académicos. Es un acto que se debe practicar en todos los ámbitos de la vida para que las personas puedan trabajar para hacer efectiva su inclusión en cualquier lugar. En este sentido, para los alumnos Sordos que estudian en las UTyP sería importante que pudieran comenzar o reforzar estas prácticas en espacios seguros, abiertos y democráticos, como pueden ser sus universidades, rescatando siempre que lo que se decida en el ámbito de las acciones inclusivas los afecta directamente. Taysum (2012) refuerza esta idea planteando que las universidades pueden ser un lugar donde los estudiantes pueden probar el fracaso y ser resilientes a él. Se sabe que en nuestro contexto la participación en los niveles más altos es muy compleja: la corrupción y la simulación de democracia se encuentra en casi todos los espacios, por lo que es menester que los jóvenes estudiantes comiencen practicando incidencia a lo largo de su trayectoria académica, para que adquieran las habilidades necesarias para la construcción de un mundo más democrático e inclusivo. Si las universidades y las y los estudiantes con discapacidad inscritos en ellas 
toman en cuenta la implicación que tiene participar, se puede evitar que deleguen a otras personas las decisiones que corresponden a elementos de vital importancia que les afectan directamente, ya sea por su falta de información o desinterés, o bien que pierdan la capacidad de opinar y estén dispuestos a someterse a prescripciones ajenas que los minimicen y no apelen al desarrollo de su autonomía (Yarza, Rojas y López, 2014).

Se puede promover entonces el protagonismo de las personas con discapacidad auditiva a través de su participación en los modelos de inclusión que se proponen en las universidades donde estudian. De tal modo que la soberanía y la creatividad de este grupo se consideran como agentes activos en la lucha para mejorar sus condiciones de vida (Corona, y Morfín, 2001).

\section{Método}

En cada una de las universidades se realizaron entrevistas semiestructuradas a las coordinadoras de los programas de inclusión y a dos intérpretes de LSM que estuvieran frente a grupo, de acuerdo con la disponibilidad de horario que tuvieran. En la Universidad Tecnológica de Santa Catarina (UTSC) se tuvo una informante más, la coordinadora de intérpretes. En la Universidad Politécnica de Santa Rosa Jáuregui (UPSRJ) al momento no contaban con esta figura. Tanto a los participantes oyentes como los Sordos se les pidió consentimiento informado antes de iniciar las entrevistas, para grabar en audio o video, según correspondiera. Todos los participantes accedieron; sin embargo, no hubo tal para poner los nombres reales dentro del presente trabajo, por ello se utilizarán seudónimos, respetando las funciones que tienen dentro de los modelos.

Es importante resaltar que esta investigación se enmarca en una mirada inclusiva por lo que fue fundamental considerar la participación de los alumnos en un grupo de discusión, donde pudieran compartir experiencias y esbozar reflexiones sobre las similitudes y diferencias que viven en las universidades públicas de México y la posible incidencia que podrían tener en los retos que se les presentan. Para ello se realizó un grupo de discusión con los alumnos Sordos participantes.

Para establecer claridad en los conceptos metodológicos, se plantea que se entienden las entrevistas como "un proceso comunicativo por el cual un investigador extrae una información de una persona...que se halla contenida en la biografía de ese interlocutor; entendiendo biografía como el conjunto de las representaciones asociadas a los acontecimientos vividos por el entrevistado" (Alonso, 199, p.226). Es una técnica que permitirá la expresión individual de un proceso socializado, es decir que podremos obtener los hábitos lingüísticos y sociales -en tanto que sistemas de esquemas generadores de 
prácticas y, al mismo tiempo, de percepciones de estas prácticas (Bourdieu, 1991, p.91, citado en Alonso, 1995 p. 227).

Por otro lado, se establece como grupo de discusión a la conversación grupal que se estructura en un espacio:

Donde el hablar está orientado al cruce, a la combinación, al lugar común donde se cruzan las diversas perspectivas y por donde todos pasan... aun proviniendo de caminos diversos... Este grupo produce un discurso donde el habla investigada puede reflexionarse y formularse preguntas... además prevalece el componente tópico de la opinión pública: las verdades cantadas como tales, lo que va de boca en boca (Canales y Binimelis, 1994 p. 111).

Pregunta de investigación: ¿Cuál es el nivel de participación, de acuerdo con la escalera de Arnstein (1969), de los alumnos sordos señantes en las Universidades Tecnológicas y Politécnicas?

Objetivo: Analizar la forma en la que los estudiantes sordos señantes participan en las UTyP en las que se encuentran inscritos.

Participantes: Los participantes de esta investigación se eligieron estratégicamente, de dos universidades de las diez en las que se encontraron alumnos sordos señantes con algún tipo de interpretación de clases en Lengua de Señas Mexicana, ya sea de tiempo completo, medio tiempo o por horas. La primera es la Universidad tecnológica de Santa Catarina en el estado de Nuevo León, que es reconocida a nivel nacional por su experiencia en inclusión educativa. Tiene más de diez años implementando el modelo de inclusión y actualmente realiza diplomados y cursos para que otras universidades puedan tomar el modelo y ser incluyentes. La segunda es la Universidad Politécnica de Santa Rosa Jáuregui, localizada en el estado de Querétaro, que no obstante su corto tiempo haciendo inclusión (3 años al momento de la investigación), es la tercera con más alumnos Sordos.

Así, tenemos que las universidades son una muestra tomada intencionalmente por las características que se buscaban cumplir para los objetivos de la investigación: que tuviera alumnos Sordos señantes, intérpretes de LSM de tiempo completo y acciones institucionales, sociales y pedagógicas que abonaran a los procesos de inclusión de las personas con discapacidad en sus escuelas. En cuanto a la selección de muestra de los alumnos e intérpretes, se realizó por disponibilidad y tomando en cuenta su experiencia y disposición a participar en las entrevistas.

Con el objetivo de facilitar la lectura de este trabajo se nombrará a los intérpretes con un número y la inicial del estado en el que se encuentra la universidad a la que pertenecen, 
por ejemplo, a un intérprete de Querétaro: Intérprete 1Q, y al de Monterrey: Intérprete 1M. A las coordinadoras se les nombrará por el puesto ocupado y a los estudiantes se les dará un nombre ficticio.

1. Universidad Politécnica de Santa Rosa Jáuregui (Querétaro).

- Entrevista con la coordinadora de la UPSRJ

- Entrevista con Intérprete $1 Q$

- Entrevista con Intérprete $2 Q$

2. Universidad Tecnológica de Santa Catarina (Monterrey).

- Entrevista con la coordinadora de la UTSC.

- Entrevista con Intérprete $1 \mathrm{M}$.

- Entrevista con Intérprete 2M.

- Entrevista con coordinadora de formación de intérpretes.

3. Los alumnos con discapacidad auditiva que forman parte del grupo de discusión fueron seleccionados por las propias escuelas, de acuerdo a sus habilidades comunicativas, a su desenvolvimiento social y su independencia.

UTSC se conformó por dos mujeres y un hombre.

- Camila: estudiante de la licenciatura en Desarrollo de negocios

- Jessica: estudiante de la ingeniería en Tecnología de la información y la comunicación

- Carlos: estudiante de la ingeniería en Tecnologías la comunicación

Por el grupo de la UPSRJ, los estudiantes participantes fueron:

- Luis: estudiante de la Ingeniería en animación y efectos visuales

- Ernesto: estudiante de la Licenciatura en Terapia física.

La locación del grupo de discusión fue un salón de posgrado en el edificio central de la UAM-X, estuvo una persona como camarógrafo, pendiente en todo momento de que la cámara estuviera grabando correctamente; había iluminación blanca y la disposición del espacio era en forma de mesa redonda. La comunicación en el grupo de discusión fue a través de la LSM, lo que permitió que los alumnos pudieran expresarse en su lengua materna de manera fluida. La moderadora, quien escribe este artículo, tiene dominio de la LSM con un nivel de interpretación educativa; además, los alumnos y alumnas estuvieron en contacto con ella semanas antes en su visita a las universidades y un día antes a su llegada a la UAM$X$. Esto permitió un acoplamiento a las formas de señar de los participantes del grupo de discusión, lo que llevó a una comunicación efectiva. 


\section{Resultados}

Este apartado está compuesto con las respuestas que surgieron según el tipo de participación que tienen los alumnos sordos dentro de las universidades, centrando la atención en la forma de involucrarse en el modelo de inclusión. Las preguntas estuvieron encaminadas a saber si los alumnos toman decisiones, si solo se les consulta, si se valora su experiencia como personas en condición de discapacidad para incidir en lo que les afectan directamente; y si consideran que las prácticas y espacios de participación llevan a los alumnos a valorarse positivamente y a considerarse agentes de cambio.

Se presentarán los resultados de las entrevistas de acuerdo con la dimensión de la participación para valorar en qué medida los alumnos y alumnas están participando en las universidades.

\section{Manipulación y utilización}

Los resultados para esta dimensión están ligados con experiencias que ha pasado la UTSC, así como con la visión que se tiene sobre la forma en que motivan a los alumnos a participar.

Durante la entrevista se comentó cómo se eligió a algunos alumnos para que representaran a la universidad en una Jornada sobre inclusión educativa. La coordinadora de la UTSC refiere que los eligieron porque "no se ponen nerviosos, son personas que pueden improvisar... que no se ajustan a un discurso, hay otros sordos que lo que les prepares dicen, pero no pueden improvisar, si hacen preguntas o algo, se quedan what?". Comentó que generalmente los estudiantes van acompañados de algún intérprete de la escuela, lo que a ella le da más seguridad. Sin embargo, esta vez se les solicitó que fueran sin acompañante, por ello eligieron a los que pensaban que eran más independientes. Estas afirmaciones y peticiones tienen que ver directamente con la dimensión de la manipulación, al expresar que se les da un discurso preparado o que se acostumbra a que vayan con intérpretes, para que las autoridades de la universidad tengan más seguridad, deja entrever en primer lugar, que no confían en las actuales habilidades de los alumnos para eventos públicos y, en segundo, que no hay un objetivo que contemple el desarrollo de estas.

Por otro lado, durante la entrevista se hipotetizó sobre el peligro de desaparición del programa de inclusión debido al cambio de rector, que ha sido su principal impulsor y puesto que los programas de inclusión no están registrados en el organigrama ni en la política pública, carecen de respaldo institucional permanente. Esto los ubica en una situación de vulnerabilidad al depender de las voluntades personales. A este respecto, la coordinadora de la UTSC comentó: 
Creemos que el programa está bastante fuerte, bastante consolidado para que cualquier otro rector que venga con ganas de ponerle atención va a funcionar, porque hay mucho personal comprometido, hay mucho alumnado; sus papás, si quisieran cancelar esto, yo creo que van y tapan la carretera y no sé qué más.

En el párrafo anterior se deja ver que, desde la percepción de la coordinadora de la UTSC, quiénes participarían manifestándose activamente serían las familias, poniéndolas a ellas y al personal comprometido de la universidad como principales actores políticos en caso de que el modelo de inclusión se pusiera en riesgo, dejando a los alumnos, por omisión, en una postura pasiva.

Lo anterior no es concluyente, son destellos que hacen pensar en los temas pendientes a abordarse en la educación inclusiva, la ausencia de programas, reflexiones y discursos sobre el tema de la participación son un indicador de que poco se ha trabajado la importancia de este aspecto fundamental para una inclusión educativa efectiva.

\section{Información, consulta y cooperación}

En este apartado, las preguntas fueron encaminadas hacia los cambios que ha tenido el programa de inclusión en cada una de las universidades y cuáles han sido las situaciones que han motivado estos cambios, como también indagar si la participación de las y los alumnos Sordos ha tenido una relación directa con esto, o si las transformaciones vienen más a partir de la observación que de la comunicación.

En este sentido, la postura de las coordinaciones corresponde a que la contribución de los estudiantes está en la evaluación del modelo, a través de encuestas que propone la misma oficina de inclusión, o bien, a través de la supervisión de ésta a las aulas de manera regular. La coordinadora de la UPSRJ comenta:

Todo el tiempo en realidad, yo paso a los salones, platico con ellos, no los estoy incitando a díganme qué está bien y qué mal, sino más bien cómo se sienten. - ¿Y sus quejas?--, pues no me ha tocado, en realidad es: oye con este maestro tenemos problemas porque no nos entiende o queremos entregar después, o tengo examen pero no me avisó... como tal ellos no han opinado mucho al respecto, pienso que ellos ya se imaginaban cómo iba a ser, creo que hemos trabajado diferente a como ellos estaban acostumbrados y eso les ha gustado.

Desde dentro de las aulas, lo que se intenta es que los y las estudiantes tengan motivación a participar dentro de la clase de manera activa, no solo en el aspecto educativo, también en el reconocimiento y reclamo de las necesidades que presentan.

Uno de los intérpretes de la UPSRJ comenta:

2Q: Yo a veces lo hablaba con el Sordo, decirle: oye por qué no le dices al maestro que la clase no te está funcionando así, y el sordo, jay es que me da pena! Entonces pues ya tenía que hablarlo yo como intérprete. 
En este sentido, se entiende que hay información, consulta y cooperación, porque los Sordos y Sordas no son ajenos a lo que está sucediendo a su alrededor, es decir, hay un intérprete que fomenta la participación, que explica la importancia de ella, pero hay situaciones personales o grupales que no permiten que esta se desarrolle. Incluso el intérprete reconoce que no está bien que se les haga todo, pero en ocasiones lo han hecho porque no encuentran otra salida para cumplir con los requerimientos de las situaciones, como puede ser entregar una solicitud para una beca en tiempo y forma o dejar que pasen más clases sin que el estudiante siga entendiendo porque el formato de la clase no tiene los ajustes necesarios requeridos.

Así, este peldaño es crítico para que los procesos de participación se ejerciten puesto que, en ocasiones, las personas con discapacidad que están llegando a las universidades no reconocen la importancia de su voz en los procesos inclusivos.

\section{Participación efectiva}

En este apartado se presentan los resultados de las preguntas encaminadas a saber de qué forma se organizan los estudiantes con discapacidad auditiva en las universidades y los aportes que esto ha traído al modelo de inclusión educativa, tratando de rescatar la participación de las personas con discapacidad como agente de cambio que favorezca su condición de estudiantes y enriquezca la experiencia de generaciones venideras en las universidades.

Al hablar de la organización que tienen los alumnos y si están comunicados para participar, las dirigentes de las coordinaciones de inclusión y las intérpretes de LSM comentaron las situaciones más sobresalientes donde vieron algún tipo de aporte de los alumnos y también remarcaron que en general no se ve participación por parte de los estudiantes, debido a que las universidades les dan todo lo que necesitan. A modo de ejemplo, en una respuesta unitaria comentaron que la falta de necesidad hace que los alumnos y alumnas se sientan cómodas con la dinámica de la inclusión.

Una de las experiencias de participación más visible, y que reporta organización por parte de los alumnos de una reconocida por la institución, la comenta la coordinadora de la UTSC, al demostrar que la participación está más en función de la persona: si llega una persona participativa se dan esos procesos de cambio, aunque la institución no lo promueva directamente

Alfonso, cuando estuvo aquí hizo muchas cosas, animó a muchos sordos,..y uno de los beneficios más grandes que dejaron a la universidad esos alumnos comandados por Alfonso fue realizar en LSM las evaluaciones. Dijo: -yo propongo que las evaluaciones que traduzcan 
y yo me invento un programita para que el reactivo aparezca con un link y se mande al video en LSM, así ahorita los sordos ya entran a los exámenes dan clic y aparece el video en LSM y ya no tiene que andar el intérprete corre y corre...

\section{La Intérprete $1 \mathrm{M}$ de la UTSC comenta:}

Nos ha tocado mucho que cuando entraban las generaciones de chavos cuando no había prepa era diferente, las que están entrando ahora que ya hay prepa, enseguida dicen yo quiero esto; el empoderamiento es diferente.... lo mejor hay menos sobre protección de la familia o de los profesores a decir sí te paso, aunque no puedas porque nada más eres uno, ya tener varios compañeros en la misma condición que tú, ya te está hablando de una identidad, de que perteneces a un grupo, que tienes responsabilidades.

\section{Grupo de discusión con alumnos Sordos de la UTSC y la UPSRJ}

El grupo de discusión fue una oportunidad de generar un espacio para que los alumnos pudieran participar de manera colectiva, en donde la reflexión, el cuestionamiento, las coincidencias y las diferencias pudieran generar ánimo de organización y participación. El grupo de discusión se abrió con la pregunta ¿para ustedes qué significa la inclusión? Esta pregunta fue derivando sus propuestas hacia la escuela, lo que ellos ven que ayuda a la inclusión en el día a día; lo que han propuesto para que mejore, ellos u otros alumnos; lo que quisieran pedir a otras instituciones y las barreras en cuanto a su participación y organización.

Lo primero en lo que los cinco participantes coincidieron es que la sensibilización es uno de los puntos más importantes para que la inclusión pueda darse de manera efectiva. De acuerdo con sus testimonios, la sensibilización tiene que ver, además de con la empatía, con que ellos puedan estar al "nivel" de los oyentes; es decir que, para ellos, su desempeño es una condición para recibir educación a nivel superior, lo que, en términos de distintos acuerdos internacionales, como la Convención, sería más bien un derecho.

En este mismo hilo conductor, Luis comenta que la sensibilización no tendría que ser únicamente con los compañeros, también los funcionarios públicos son pieza clave. Los comentarios de este estudiante son sumamente importantes porque puntualizan uno de los problemas medulares de los programas de inclusión en México: que las acciones estén vinculadas a la buena voluntad de las personas que los inician y que no existan en el organigrama, ni que tengan presupuesto autónomo para seguir operando independientemente de la persona que esté a cargo de las universidades, obliga a que todos los directivos estén sensibilizados para que los programas sobrevivan y para que puedan replicarse en otras universidades.

Una vez que salió la idea de solicitar que de alguna manera se reproduzcan los modelos de inclusión en otras instituciones, los alumnos comenzaron a describir los procesos 
de participación en los que habían estado. Con una pregunta más por parte de la moderadora, propusieron la forma en que podrían organizarse para realizar sus peticiones.

Luis comenta:

Ustedes de la UTSC, los de la UPSRJ, los de la UAM, cada uno debería escoger a un líder donde primero se discutan los proyectos, lo que hace falta, la idea es que cada uno comparta su opinión, que se haga un listado de lo que se necesita y se lleve al gobierno, a Peña Nieto, deberíamos hacer unión.

Otro punto que se tocó al comentar la participación que han tenido en sus escuelas está directamente relacionado con las vivencias y barreras con las que se topan. Un ejemplo de ello es lo que Camila comenta:

en $2014 \ldots$ nos estaban explicando algo de negocios, pero muy profundo, muchas palabras... y yo con mi cara de (signo de interrogación) llegó la hora en que nos dijeron hagan la actividad... y pensé, ¿cómo le puedo hacer? Entonces, cree un proyecto que se llama Glosario (en LSM)... de esa manera cree una herramienta para no estar teniendo que molestar a los intérpretes o a los oyentes.

La aplicación que desarrolló esta alumna es un gran paso para que los neologismos en LSM sean accesibles a las personas Sordas y se puedan ir compartiendo a las próximas generaciones.

Aunque existe una barrera: no siempre en las universidades que hay inclusión existe la carrera que ellos desean estudiar. Lo que opinan a este respecto es que mientras que no se abran todas las carreras en LSM, los Sordos deberán unirse y estudiar algo, aunque no sea completamente de su agrado; de esta forma compartieron que estarán apoyando a la inclusión, pues promoverán que se abran espacios para su comunidad.

Cuando se planteó la pregunta ¿cómo apoyarían para que más Sordos pudieran estudiar en las universidades?, se pudo observar que para ellos es verdaderamente importante seguirse superando académicamente para demostrar que los Sordos pueden y que esto los hace acreedores a un lugar en las universidades. Parece que cuando dicen en repetidas ocasiones "que copien el modelo" quieren decir que los encargados de eso no son ellos, sino "alguien" institución o persona ajena a este grupo, quienes deberían encargarse de implementar programas de inclusión. De acuerdo con su perspectiva, una forma en que ellos pueden aportar es pasando de boca en boca el éxito del programa de inclusión al que se encuentran inscritos. Una participante hace eco con un comentario que puede vislumbrarse como una razón de por qué se viven desvinculados, Camila:

¿yo te pregunto, son las mismas señas en toda la república? Supongamos que yo me grabo y difundo el video, algunos Sordos comenzarán a criticarme, que esa seña está mal, que no están de acuerdo, entonces todos nos empezamos a criticar y a pelear, a estar en contra unos con otros. Yo lo hice para la escuela, ese era mi objetivo y ya. 
Ernesto aporta: "es que todos se ignoran, yo los he invitado a juntas y me ignoran".

Ante esta situación Camila propone: “... no pasárselo a todos, pero, por ejemplo, entre estas tres universidades, nosotros que estamos de acuerdo; por qué no acordar nosotros y hacerlo entre nosotros. Y en el futuro podríamos defendernos, trabajar, comunicarnos". Otra idea que da Luis es: "que hoy sea la primera reunión y que la próxima venga más gente y que podamos compartir, y que haya la tercera, la cuarta... pero tú (haciendo alusión a la moderadora) nos vas a tener que pagar (los viáticos)".

Hasta aquí se puede ver que los alumnos y alumnas participan de manera efectiva en cosas que les competen directamente a ellos, como el caso de Camila, que creó un glosario y como efecto secundario pudo impactar a otros compañeros. No es su deseo o ímpetu generar respuestas a las problemáticas de su comunidad que pueden vislumbrar; para ellos es más importante mantenerse competitivos y demostrar con hechos que ellos "aunque" sean Sordos pueden terminar una carrera universitaria. También es importante resaltar que se sienten afortunados por tener lo que tienen lo que los mantiene en un lugar de "quedarse tranquilos" como dijo Jessica, como una muestra de agradecimiento a los servicios de inclusión que se les ofrecen.

\section{Discusión}

El aporte de este trabajo deja ver que el tema de participación no es algo que se piense dentro de las universidades. Queda pendiente indagar si es debido a que no lo consideran parte de sus objetivos formativos, si dan por hecho que lo hará quien lo necesite, o si definitivamente no es algo que han pensado que es importante trabajar para el mejor desarrollo de las personas con discapacidad.

Es por esta razón que toma como necesario que la implementación de programas inclusivos sea desde la educación básica, un motor para generar espacios más democráticos. Es importante reconocer que las escuelas que hoy, en México, dan el paso de reconocer y hacer valer el derecho de las personas con discapacidad a estar en una comunidad educativa, lo hacen de acuerdo con sus recursos humanos, económicos e intelectuales, por lo que la mejor manera de hacer frente a la complejidad de implementar modelos inclusivos es con la ayuda mutua, la interdependencia y las redes de colaboración (Echeita, 2008).

Una de las principales áreas de oportunidad encontradas en este estudio es que las universidades no están en contacto, no tienen claridad de cómo están haciendo la inclusión en otros espacios, los alumnos están incomunicados interinstitucionalmente, lo que en ocasiones hace que sus aportes no sean llevados a otras comunidades educativas para 
mejorar su situación académica. Por ello se quiere presentar como propuesta aportes de otros estudios donde la participación de los alumnos en la educación básica es menester para crear las condiciones de espacios inclusivos. Sería imperativo reconocer en el alumnado su posibilidad de participar en los intentos de mejora, como agente de cambio y transformación en los modelos de inclusión, ya que son estos los que, de acuerdo con (Rojas, Haya y LázaroVisa, 2011), han caracterizado los procesos de cambio o innovación educativa, como en la UTSC, con el proyecto de los exámenes en video en LSM.

De acuerdo con este mismo autor, los proyectos en donde los alumnos participan y su voz es escuchada, les permiten desarrollar el sentido de la "responsabilidad o de la autoría y el del empoderamiento respecto a lo que ocurre a su alrededor, lo que sin duda contribuye a su preparación como ciudadanos presentes y futuros de una sociedad democrática" (Rojas, Haya y Lázaro-Visa, 2011 p.97).

Finalmente, se pone a discusión el objetivo de las universidades al realizar educación inclusiva; ¿se piensa como objetivo final que los alumnos y alumnas con discapacidad tengan un trabajo? o bien ¿se está apostando a que estos modelos puedan ser formadores de agentes de cambio que impacten no sólo en ellos mismos sino en las generaciones venideras? (Rosano, 2013); plantea que la escuela inclusiva, democrática, no puede ser la que se centre en la competencia, debería ser la que participe para compartir no para competir.

\section{Conclusiones}

A partir de los aportes de los informantes, se pueden distinguir al menos cuatro aspectos relevantes que influyen en la participación de las personas con discapacidad auditiva en los modelos de inclusión de sus universidades.

1. La disposición personal que cada alumno o alumna pueda traer de acuerdo con su contexto educativo, cultural y familiar; casos de estudiantes que, aunque no se les motive, son agentes de cambio y generan propuestas que les benefician a ellos y a su comunidad, a partir de su experiencia como personas en condición de discapacidad y del conocer en profundidad sus necesidades, y producir así estrategias de intervención innovadoras.

2. Es probable que, si están involucrados en asociaciones civiles o con otros centros educativos, deportivos o políticos, tengan más incidencia dentro de la universidad, generando vínculos entre esta y las organizaciones que trabajen temas que sean del interés de los alumnos.

3. Ambas universidades hacen alusión a la importancia de la actitud participativa de las generaciones más recientes, debido a que crecen en número y en experiencia. Esto permite que se sientan con más fuerza para pedir los cambios que necesitan con los profesores y directivos. 
4. Finalmente, el Intérprete $2 Q$ da una pista de por qué los profesionistas involucrados con los alumnos no promueven la participación:

Pues ellos fuera de la universidad sí se organizan, pero dentro no fomentamos la participación porque a la hora de la salida lo que ya quieres es descansar y algo así sería voluntario...

La propuesta gira, entonces, sobre asumir la investigación como el centro a través del cual la participación de los estudiantes debería jugar un papel fundamental. En el grupo de discusión, los aportes que los alumnos pusieron sobre la mesa, reconociendo su participación de forma activa, estaban enmarcados en lo académico más que en la acción asociativa o política; también mostraron inquietud por encontrar formas de comunicarse con otras instituciones para compartir sus experiencias y que los modelos se "replicaran".

Por otra parte, sería pertinente reflexionar sobre la importancia de dar a las personas con discapacidad auditiva una materia que hable de su comunidad, de la historia de la educación de los Sordos y de cómo han ido cambiando las perspectivas de esta. Para que los alumnos puedan participar, es necesario que estén informados sobre su historia y los agentes que han posibilitado estos cambios. De esta manera, podrían fortalecer su autoconfianza y la toma de decisiones basada en información vinculándola con superación de barreras de manera colectiva.

Así, la educación inclusiva debe abonar no únicamente a que los alumnos puedan conseguir un trabajo al terminar su educación. Si bien es cierto que la independencia económica es un objetivo importante y que las universidades lo tienen claro, se debe ver la formación como un proceso integral y no sólo como académico-laboral.

En este sentido la Observación que hace Naciones Unidas sobre el derecho a la educación inclusiva en 2016, apunta que en los planes de estudio se deben tomar en cuenta temas de ciudadanía y capacidades de autopromoción y autorrepresentación como base de la participación política y social.

Además, al reforzar la idea de la potencialización de sus propuestas y proyectos donde exponen sus vivencias y experiencias académicas, este informe plantea que estas medidas deben garantizar su derecho al enriquecimiento propio y de la sociedad.

Para terminar, propongo que las universidades incluyentes sean un espacio que promueva en los estudiantes la idea de que son agentes de cambio. De acuerdo con la literatura, la forma de llegar a ello es que los alumnos vivan experiencias significativas en temas que les sean de interés. En este sentido, las instituciones deben brindar información para que se abran los debates de las aportaciones que ellos pueden hacer. La literatura menciona que un problema a enfrentar en las sociedades modernas occidentalizadas es que 
los espacios públicos se han colonizado por la lengua y cultura hegemónica, lo que deja a los Sordos en un espacio complicado para identificar aquello que les resulta valioso (Tubino, 2009;2012).

Así podemos decir que un agente es "alguien que actúa y produce cambios" (Sen, citado en Naciones Unidas, 2011), modificando la ruta previsible de la historia. Esta acción se da dentro de los límites de lo instituido, entonces este agente podrá tomar iniciativas para generar procesos que innoven a partir de la crítica del ahora para mejorar el futuro.

\section{Referencias bibliográficas}

Alonso, L (1995). Sujeto y discurso: El lugar de la entrevista abierta en las prácticas de la sociología cualitativa, en Delgado, J y Gutiérrez, J (Comp). Métodos y técnicas cualitativas de investigación en ciencias sociales. (pp.: 225-240). España: Síntesis.

Arnstein, S. (1969). A ladder of citizen participation. AIP Journal, Julio, 216-224.

Barnes, C. (2007). Disability Studies: What's the Point? Intersticios: Revista Sociológica de Pensamiento Crítico, 1 (1), 1-10. Recuperado de: www.intersticios.es/article/download/673/549

Canales Cerón, M., y Binimelis Sáez, A. (1994). El grupo de discusión. Revista de Sociología, 0 (9). 107-119. Recuperado de http://www.revistadesociologia.uchile.cl/index.php/RDS/article/view/27647/29314

Castilla, M. e Inciarte, A. (2004). La naturaleza de la acción participativa y la formación para participar. Espacio Abierto, 13 (2), abril-junio, 249-275.

Corona, Y. y Morfín, S. (2001). Diálogo de saberes sobre participación infantil. UAM UNICEF, Comexani, Ayuda en Acción México.

Coster, W., Bendell G., Lijienquist, K., Kao, Y-C., Khetani, M. y Teplicky, R., (2013). School participation, supports and Barriers of students with and without disabilities. Child care, health and development, 39 (4), 535-543.

Echeita Sarrionandia, G. (2008). Inclusión y Exclusión Educativa “Voz y Quebranto”. Revista Electrónica Iberoamericana sobre Calidad, Eficacia y Cambio en Educación, 6 (2), 9-18.

Expósito, L (2014). Participación adolescente en la secundaria: explorando lo deseable, lo potencial y lo permisible. Argumentos, Procesos educativos y dinámicas sociales contemporáneas Dossier, 27 (74), 47-74. 
Expósito, L. (2015). Scope and Quality of Student Participation in School: Towards an Analytical Framework for Adolescents. International Journal of Adolescence and Youth, 20(3), 346-374 DOI: 10.1080/02673843.2015.1009920 http://dx.doi.org/10.1080/02673843.2015.1009920

Gómez, R. (2014). Inclusión de la persona Sorda a la Educación Superior. México: Revista Latinoamericana de Educación Inclusiva, 8 (1), marzo-agosto, 93-108.

Hart, R. (1993). La participación de los niños: de una participación simbólica a una participación auténtica, en Ensayos Innocenti (4). UNICEF.

Joly, E. ¿Qué les pasa a la ciencia, a la tecnología y a la universidad con relación a las personas con discapacidad? ¿Las ven, las escuchan, o son sordas y ciegas? En Katz, L. y Danel, P. (Comp). (2011). Hacia una universidad accesible: Construcciones colectivas por la discapacidad. (pp.: 43-64). La Plata: Universidad Nacional de La Plata.

London, S; Formichella, M (2006) El concepto de desarrollo de Sen y su vinculación con la Educación Economía y Sociedad, XI (17), 17-32.

López, M., López, M. y Llorent, V. (Coord). (2009). La Discapacidad: Aspectos Educativos y Sociales. Málaga España: Aljibe.

Oliver, M. (1990). The Politics of Disablement. Basingstoke: Macmillan Press.

Rodríguez, M., Alvarado, A. y Moreno, M. (2007). Construcción participativa de un modelo socioecológico de inclusión social para personas en situación de discapacidad. Acta Colombiana de Psicología, 10 (2), 181-189.

Rojas Pernía, S., Haya Salmón, I., Lázaro-Visa, S. (2012). La voz del alumnado en la mejora escolar: niños y niñas como investigadores e Educación Primaria. Revista de educación (359), 81-101.

Rosano, S. (2013). "Son cosas de niños" La participación como derecho y la educación inclusiva: reflexión en torno al papel de las niñas y niños en la escuela. Revista latinoamericana de Educación Inclusiva, 7 (1), 151-167.

Ruíz, M. y Cruz, M. (2013). Derecho a la Educación. En Educación, desigualdad y alternativas de inclusión 2002-2012 (pp.: 47-92). Colección Estados del Conocimiento. México: ANUIES- Consejo Mexicano de Investigación Educativa.

Taysum, A. (2013). The Impact of Doctoral Study on Educational Leaders' work for Students' participation in Education Systems and Society. Educational Review, 65 (4), 432-446, http://dx.doi.org/10.1080/00131911.2012.708020 
Torres, S. (1995). Deficiencia auditiva: Aspectos Psicoevolutivos y educativos. Málaga: Aljibe.

Tubino, F (2009/2012). Libertad de Agencia: entre Sen y H. Arendt. Primera reimpresión en Desarrollo humano y libertades. Perú: Pontificia Universidad Católica.

Yarza, A., Rojas, H. y López, J. (2014). Discapacidad y educación superior: las contiendas por la participación y la ciudadanía en las reformas neoliberales de la Universidad y la educación superior en Colombia. Revista Latinoamericana de Educación Inclusiva, 8, (1), 35-49.

\section{Otros documentos consultados:}

Consejo Nacional para Prevenir la Discriminación. Convención sobre los derechos de las personas con discapacidad. (2006). CONAPRED, México.

Naciones Unidas, (2011). Agencia y empoderamiento en la medición de la pobreza. Revista latinoamericana de desarrollo humano. Boletín Nro. 79, 1-17. 